\title{
THERMOHYDRODYNAMIC ANALYSIS OF A JOURNAL BEARING WITH A MICROGROOVE ON THE SHAFT
}

\section{SAMUEL CUPILLARD}

Hydro-Québec Research Institute

Varennes, QC, Canada

cupillard.samuel@ireq.ca

\section{MICHEL J. CERVANTES}

Luleå University of Technology, Division of Fluid Mechanics

Luleå SE-971 87, Sweden

\section{SERGEI GLAVATSKIH}

Royal Institute of Technology, Division of Systems and Component Design

Stockholm SE-100 44, Sweden

\begin{abstract}
In this study, thermohydrodynamic performance of a journal bearing with a microgroove created on the shaft is analysed. A plain journal bearing is modelled using a computational fluid dynamics (CFD) software package. Navier-Stokes and energy equations are solved. The rotor-stator interaction is treated by using a computational grid deformation technique. Results are presented in terms of typical bearing parameters as well as flow patterns. Results are also compared to the bearing with a smooth shaft.

The effect induced by a microgroove on pressure distribution is explained for different bearing configurations, eccentricities and microgroove depths. It is shown that the microgroove produces a local drop in pressure which, averaged over one revolution, decreases the load carrying capacity. The load carrying capacity is further decreased by using deeper microgrooves. With thermal effects considered, the microgroove carries more cold lubricant into the warmest regions of the bearing. This effect, more pronounced with deeper microgrooves, is due to a global flow recirculation inside the microgroove, which improve mixing.
\end{abstract}

\section{INTRODUCTION}

Texturing applied on one of the surfaces in a hydrodynamic contact has been widely studied over the past years. Most of the studies focused on textures on the stationary surface and only a few studies were devoted to texture located on the moving surface. In an experimental study, Snegovskii and Arnautova (1983) reported that dimples machined on the shaft surface in a journal bearing allowed an increase in load carrying capacity by a factor of 1.5 to 2 and a reduction of frictional losses by 10 to $15 \%$ at high sliding speeds (30 to $60 \mathrm{~m} / \mathrm{s}$ ). However, in the low sliding speed range ( 5 to $10 \mathrm{~m} / \mathrm{s}$ ), the load carrying capacity was reduced by a factor of 1.3 to 1.6. Winoto et al. (2002) tested herringbone-groove patterns on the shaft of a journal bearing. The performance in terms of pumping sealing and stiffness were concluded as promising especially for well chosen groove length ratios. Kobayashi (1999) investigated the steady state performance and dynamic stability of a herringbone-grooved journal bearing with a compressible Reynolds equation. As the shaft texture is not aligned with the coordinate axis, a coordinate mapping was used to account for this. The Reynolds equation was solved in a modified coordinate system. A similar transformation of the coordinate system is presented in the work of Jang and Chang (2000) who recommended to consider 
cavitation in the modelling of such herringbone-grooved journal bearings. They also pointed out that herringbone grooves result in a smaller value of pressure with a wavy distribution compared with the pressure developed for a plain journal bearing. Isothermal conditions were assumed in these two studies (Kobayashi 1999, Jang and Chang 2000).

The mechanism enhancing load carrying capacity of hydrodynamic bearings with a partially textured stator was explained by Cupillard et al. (2008a). Texture effect on the load carrying capacity may be positive for a low convergence ratio or low eccentricity, otherwise it is negative. The effect of a partially textured rotor has not yet been elucidated.

The goal of this study is to investigate the effect of a partially textured rotor considering a single microgroove. A CFD analysis is conducted for a laminar and unsteady flow in a journal bearing. A two dimensional analysis is first performed to understand the effect of the microgroove and validate numerical approach. Energy equation is then taken into account as well as cavitation in order to correctly handle the pressure in the divergent part of a three dimensional bearing at high eccentricities. A comparative analysis of the results obtained for smooth and microgrooved shafts for different eccentricities and groove depths is carried out.

\section{NUMERICAL MODEL}

\section{Geometry and boundary conditions}

Both a 2D and 3D journal bearing models are used in this work, see Figure 1. A symmetry condition is applied at the midplane of the bearing to simulate only half of the domain. Bearing dimensions are as follow: shaft radius $R_{S}=0.05 \mathrm{~m}$, radial clearance $\mathrm{c}=145 \mu \mathrm{m}$, half bearing length $\mathrm{L}_{\mathrm{z}}=0.0665 \mathrm{~m}$, side channel extent in radial direction $\mathrm{R}_{\mathrm{c}}=0.1 \cdot \mathrm{R}_{\mathrm{S}}$ and axial direction $\mathrm{L}_{\mathrm{c}}=0.1 \cdot \mathrm{L}_{\mathrm{z}}$. The shaft angular speed is set to $\omega=48.1 \mathrm{rad} / \mathrm{s}$.
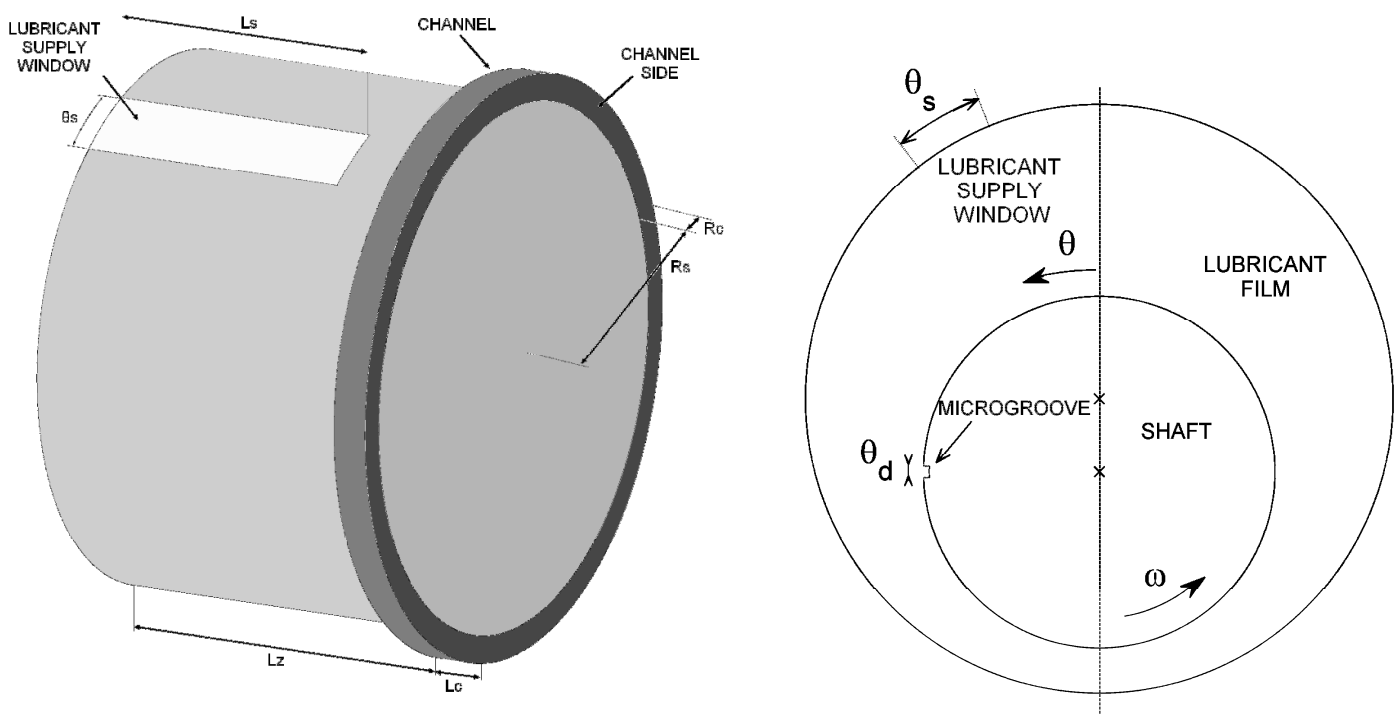

Figure 1: Computational domain (left) and its cross section in the midplane (right) where the microgroove is located at $\alpha=90^{\circ}$. The size of radial clearance is magnified.

At the bearing and shaft surfaces, adiabatic and no-slip boundary conditions are assumed. A source of cold lubricant is considered on a window of the bearing surface defined at an angular location $\left(\Phi=30^{\circ}\right)$, angular extent $\left(\theta_{\mathrm{s}}=16^{\circ}\right)$ and length $\left(\mathrm{L}_{\mathrm{s}}=0.8 \cdot \mathrm{L}_{\mathrm{z}}\right)$. A mass flow is imposed at the supply window and the velocity of the lubricant is determined by flow continuity. The computational domain is extended by a channel on the bearing side and the 
boundary is moved away in order to avoid setting a temperature at this location. At the side of this channel, the lubricant is allowed to flow in and out of the domain and relative pressure is set to be zero. The bearing is submerged in a constant temperature oil bath at the same value as the inlet temperature $\mathrm{T}_{\text {in }}=35^{\circ} \mathrm{C}$. The lubricant has a temperature dependent viscosity and specific heat. The $2 \mathrm{D}$ or infinitely long bearing is a model restricting the domain to the midplane. In this case, isothermal conditions are imposed, no supply window is considered and the dynamic viscosity, 0.0127 Pas, is constant.

A single texture cell, a microgroove, located on the shaft is investigated while the bearing surface is smooth. The 2D microgroove and the cross-section of the $3 \mathrm{D}$ microgroove are taken as rectangular, see Figure 1. The microgroove is characterized by its circumferential width $\left(\theta_{\mathrm{d}}=4^{\circ}\right)$, depth $(\mathrm{d}=\{0.5,1,2,4\} \cdot \mathrm{c})$ and length $\left(\mathrm{l}=0.5 \cdot \mathrm{L}_{\mathrm{z}}\right)$. The dimensions are taken similar to the microgrooves analysed in a previous study (Cupillard et al. 2008b) where it was shown that increasing the microgroove axial length decreased the load carrying capacity. Therefore the microgroove should not be chosen too long in the axial direction, but also not too short as the longest grooves are believed to have a better pumping and cooling effect.

\section{Equations}

The code used in the analysis is CFX 11.0. The Navier-Stokes equations, momentum equation (1) coupled with the continuity equation (2), are solved over the domain together with the energy equation (3), using the finite volume method. The flow is laminar and transient. Thermal effects are considered in the 3D domain but not in the $2 \mathrm{D}$ case.

$$
\begin{gathered}
\frac{\partial\left(\rho u_{i}\right)}{\partial t}+\frac{\partial\left(\rho u_{i} u_{j}\right)}{\partial x_{j}}=-\frac{\partial p}{\partial x_{i}}+\frac{\partial}{\partial x_{j}}\left(\mu\left(\frac{\partial u_{i}}{\partial x_{j}}+\frac{\partial u_{j}}{\partial x_{i}}\right)\right) \\
\frac{\partial \rho}{\partial t}+\frac{\partial\left(\rho u_{i}\right)}{\partial x_{i}}=0 \\
\frac{\partial\left(\rho h_{t o t}\right)}{\partial t}-\frac{\partial p}{\partial t}+\frac{\partial}{\partial x_{i}}\left(\rho u_{i} h_{t o t}\right)=\frac{\partial}{\partial x_{i}}\left(\lambda \frac{\partial T}{\partial x_{i}}\right)+\frac{\partial u_{i}}{\partial x_{j}}\left[\mu\left(\frac{\partial u_{i}}{\partial x_{j}}+\frac{\partial u_{j}}{\partial x_{i}}\right)-\delta_{i j}\left(\frac{2}{3} \mu \frac{\partial u_{k}}{\partial x_{k}}\right)\right]
\end{gathered}
$$

where $\delta_{\mathrm{ij}}=1$ when $\mathrm{i}=\mathrm{j}$, and 0 when $\mathrm{i} \neq \mathrm{j}$. $\mathrm{h}_{\text {tot }}$ is the total enthalpy.

The cavitation model used allows sub-ambient pressures and is based on a density-pressure relationship (Cupillard et al. 2008b). When the lubricant pressure drops below the cavitation pressure $\left(\mathrm{p}_{\mathrm{cav}}=30000 \mathrm{~Pa}\right)$, the density decreases according to the following law:

$$
\rho= \begin{cases}\rho_{0} & \text { if } p>p_{c a v} \\ \rho_{0}\left[3\left(\frac{p}{p_{c a v}}\right)^{2}-2\left(\frac{p}{p_{c a v}}\right)^{3}\right] & \text { if } p \leq p_{c a v}\end{cases}
$$

The model is applied to a single phase flow with a varying density. It has the advantage of being more stable compared to any multiphase flow cavitation model. Numerical convergence is also easier to achieve. The simulations are performed with the high resolution scheme for the advection term.

\section{Model for the motion of a microgroove on the shaft}

A grid update method loads a new grid at each time step $\Delta t$ and solves the flow equations as presented in Figure 2. The solution at a time step uses the solution at the previous time step as initial guess. The new geometry differs only in the position of the microgroove $(\alpha)$ in the circumferential direction. A routine calls the mesh files in which the coordinates of all nodes 
are explicitly sets. The topology and connectivity of the meshes remains fixed. The coordinates are read and updated. Loading the grids successively and solving the flow over each grid at the current time step enable to create a physical and realistic motion of the shaft. With enough grids loaded over one period, the rotation of the shaft with the microgroove is accurately resolved. The simulation is performed until the time $t$ reaches a pre-set total time $t_{\text {total }}$. The bearing boundary is given zero velocity whereas the shaft velocity is related to the displacement of the grid nodes. Therefore, for a given angular speed, the time step depends on the number of grids loaded over one rotation of the shaft.

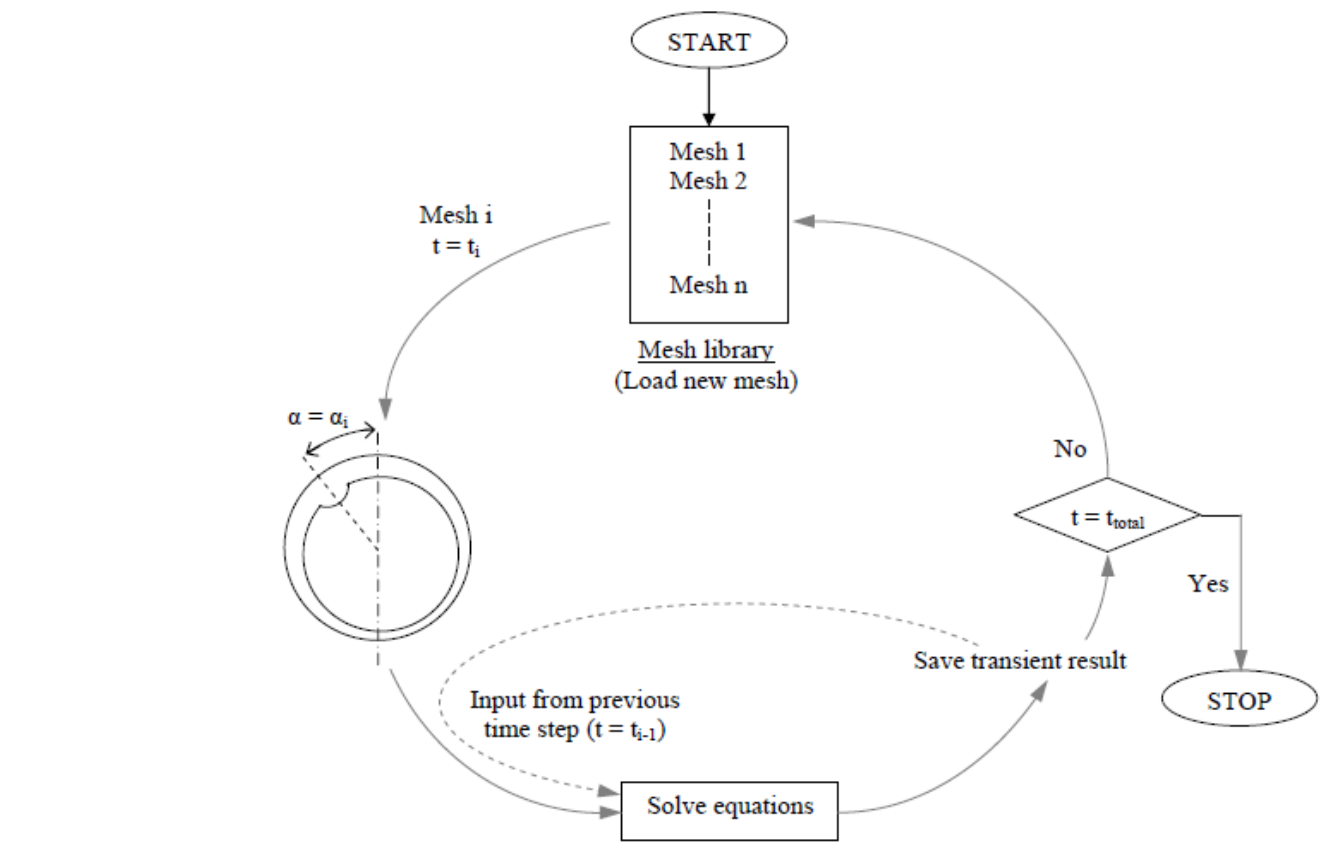

Figure 2: Flow chart of the grid update method

This method requires a significant amount of grids and thus a large disc space. The number of grids generated $n$ is defined by the desired number of positions of the microgroove in the circumferential direction. For an angular shaft speed $\omega$, the time step is $\Delta t=2 \pi /(\omega \cdot n)$. More computational effort is necessary for smaller time steps.

\section{Validation of the computational results}

For the 2D bearing, 54000 elements are used in each mesh, with 30 elements in the film and 1800 elements in the circumferential direction. For the 3D bearing, about 1 million elements are used for each of the meshes with 22 elements in the film, 1080 in the circumferential and 32 in the axial directions. In order to determine how many meshes shall be loaded over one shaft revolution to get a good accuracy, the following investigation is performed: $45,90,180$ and 360 meshes are used for a microgrooved shaft in a 2D model with $\varepsilon=0.1$, see Figure 3 . The time steps used are $\mathrm{t}_{45} \approx 2.90 \mathrm{~ms}, \mathrm{t}_{90} \approx 1.45 \mathrm{~ms}, \mathrm{t}_{180} \approx 0.73 \mathrm{~ms}$ and $\mathrm{t}_{360} \approx 0.36 \mathrm{~ms}$ respectively. For the microgrooved shaft $(\mathrm{d} / \mathrm{c}=0.5)$ the load is periodic, with a period of one shaft revolution, therefore, only one period is presented starting from zero relative time. The load value of the steady state case, obtained with a simulation using one fixed mesh and with the velocity set at the shaft boundary, is added for comparison. At the beginning and at the end of the period, the microgroove is in the maximum film region $\left(\alpha=0^{\circ}\right)$. The microgroove passes the minimum film at half period $\left(\alpha=180^{\circ}\right)$. The load variation over one revolution of the microgroove presents some differences between the cases but seems to converge. The motion of the microgrooved shaft is considered to be resolved accurately enough when the 
angular resolution of the motion is 1 degree. Therefore, the case with 360 meshes loaded over one revolution is used in this study.

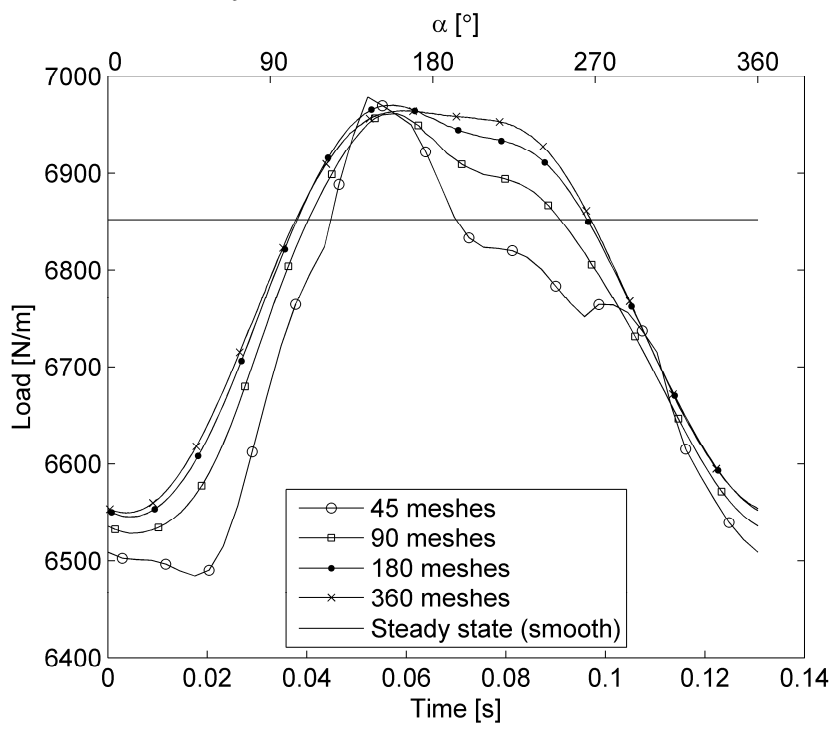

Figure 3: Variation of the load carrying capacity with time over one period, for a $2 D$ bearing with a microgrooved shaft, $d / c=0.5(\varepsilon=0.1)$

\section{RESULTS}

\section{Two dimensional bearing}

Low eccentricity ratio $(\varepsilon=0.1)$ is chosen for the 2D bearing. For this condition, cavitation is not expected and therefore not modelled. For $\mathrm{d} / \mathrm{c}=0.5$, variation in load carrying capacity is almost a sinusoid with a maximum and a minimum value over a revolution, Figure 3 . The minimum value of the load occurs when the microgroove is around the maximum film, at $t \approx$ $0.13 \mathrm{~s}$. The maximum value occurs when the microgroove is close to the minimum film, at $\mathrm{t} \approx$ $0.065 \mathrm{~s}$. These findings confirm the results of Jourak et al. (2008) that found the same trend using the general grid interface (GGI) technique. When passing the maximum film $\left(\alpha=0^{\circ}\right)$ in the positive pressure gradient region, the microgroove decreases the positive part of the pressure, see Figure 4 (left). At the minimum film $\left(\alpha=180^{\circ}\right)$ where pressure gradient is negative, the microgroove has a positive contribution to the pressure, see Figure 4 (right). The microgroove produces a drop of pressure in the stationary coordinate frame.
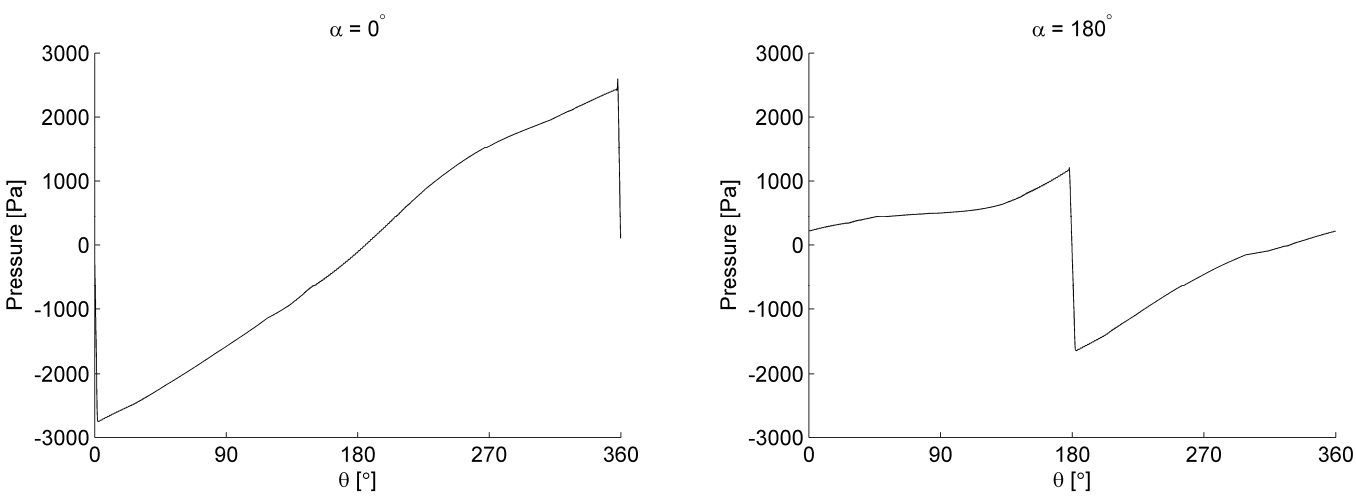

Figure 4: Pressure differences between the bearing with a microgrooved and smooth shaft, $d / c=1$ and $\varepsilon=0.1$. Pressure decreases due to the microgroove at $\alpha=0^{\circ}$ and $\alpha=180^{\circ}$. 
The microgroove sees the stator moving in the opposite direction from the rotor. The pressure in the fluid film is seen in the direction of motion of the stator by the microgroove. Therefore the effects of the microgroove regarding its location are reversed compared to a stationary texture (Cupillard et al. 2008a). The microgroove located in the region of negative pressure gradient $\left(100^{\circ}<\alpha<260^{\circ}\right)$ enhances the load carrying capacity compared to a bearing with a smooth shaft, see Figure 3. The microgroove has a negative contribution to the load carrying capacity in the region of positive pressure gradient.

A larger microgroove depth induces a loss of load carrying capacity, see Figure 5 . In the stationary coordinate frame, some recirculating flow is transported with the microgroove. The presence of recirculation in a microgroove tends to reduce local pressure gradient and decreases load carrying capacity (Cupillard et al. 2008a). This reduces the positive effects that the microgroove brings in the region of negative pressure gradient and affects the load curve. An increase in the depth of the microgroove produces then a loss in load carrying capacity when the microgroove passes the region of minimum film thickness, see Figure 5. Two maxima of the load now occur over one revolution.

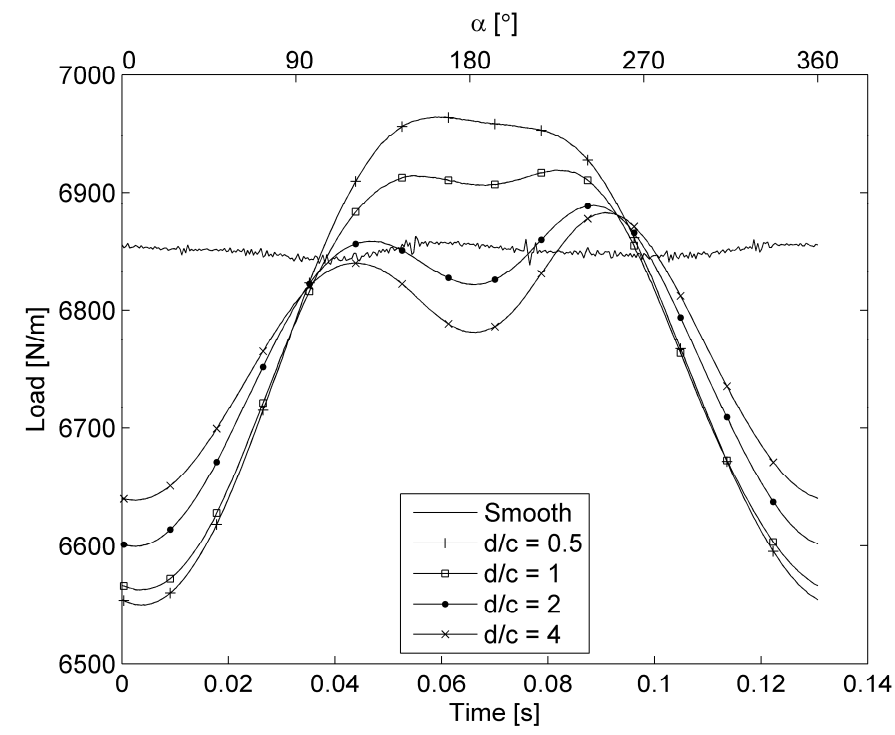

Figure 5: Influence of the microgroove depth on the load carrying capacity, $\varepsilon=0.1$

Load carrying capacity averaged over one revolution decreases as the microgroove depth increases, see Table 1. Friction is continiously reduced with the microgroove depth as the viscous losses decrease. The overall effect of the microgroove deteriorates hydrodynamic performance under isothermal conditions for a $2 \mathrm{D}$ case. The influence of the microgroove located on the shaft of a 3D bearing is investigated next considering thermal effects.

Table 1: Differences (in \%) in the load carrying capacity $(W)$, friction force $\left(F_{r}\right)$ and friction coefficient $\left(f=F_{r} / W\right)$ averaged over one revolution between the $2 D$ bearings with a microgrooved and smooth shaft, $\varepsilon=0.1$

\begin{tabular}{|c|c|c|c|}
\hline & $\Delta \mathrm{W} / \mathrm{W}_{\text {smooth }}$ & $\Delta \mathrm{F}_{\mathrm{r}} / \mathrm{F}_{\mathrm{r} \text { smooth }}$ & $\Delta \mathrm{f} / \mathrm{f}_{\text {smooth }}$ \\
\hline $\mathrm{d} / \mathrm{c}=0.5$ & -0.90 & -0.33 & +0.57 \\
\hline $\mathrm{d} / \mathrm{c}=1$ & -1.09 & -0.35 & +0.75 \\
\hline $\mathrm{d} / \mathrm{c}=2$ & -1.15 & -0.45 & +0.71 \\
\hline $\mathrm{d} / \mathrm{c}=4$ & -1.11 & -0.59 & +0.52 \\
\hline
\end{tabular}




\section{Three dimensional bearing}

A 3D bearing is simulated first with an eccentricity of 0.1 and an inlet mass flow rate of $0.001 \mathrm{~kg} / \mathrm{s}$. Then, the eccentricity ratio is increased to $\varepsilon=0.61$, the inlet mass flow is chosen to be equal to $0.01 \mathrm{~kg} / \mathrm{s}$ and cavitation is considered. As for the 2D case, 360 meshes are loaded over one shaft revolution. The simulations are started from a fully converged solution of a steady state case with the smooth shaft as an initial guess in order to have a well established temperature distribution at the start. Figure 6 shows load carrying capacity for different microgroove depths at $\varepsilon=0.1$ and 0.61 . Values for the smooth cases are also shown for comparison.

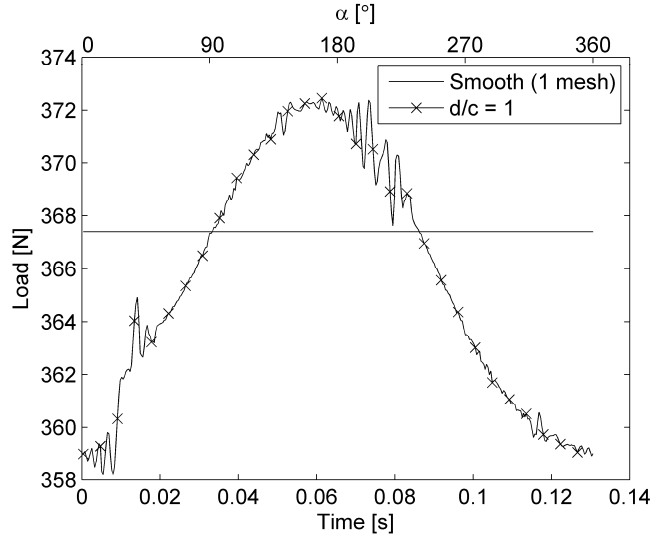

(a) $\varepsilon=0.1$

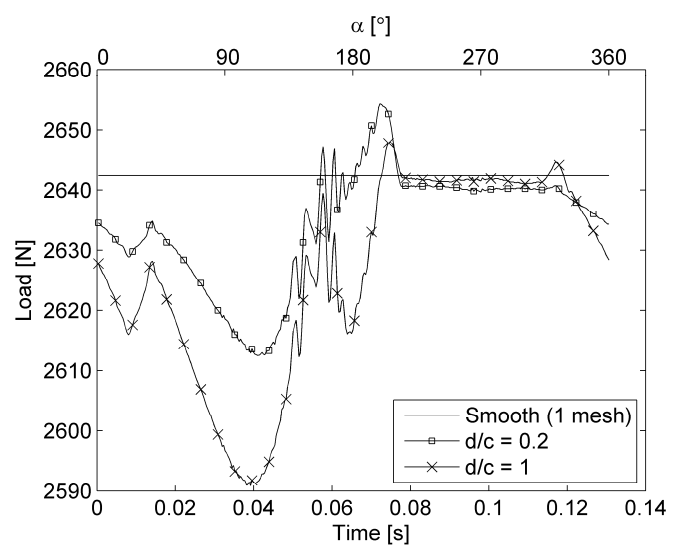

(b) $\varepsilon=0.61$

Figure 6: Load carrying capacity for different microgroove depths

For $\varepsilon=0.1$ in Figure 6(a), the load presents the same trend as for the 2D bearing with positive effects in the minimum film region followed by negative effects outside, as explained previously. For $\varepsilon=0.61$ in Figure $6(\mathrm{~b})$, the trend is different as pressure is not increased in the region of negative pressure gradient, see Figure 7 (right). Thus, the presence of the microgroove in this region leads to some loss of pressure, as explained by Cupillard et al. (2008a). Consequently, load carrying capacity is not increased when the microgroove passes the minimum film region. The decrease of pressure and load carrying capacity becomes largest when the microgroove is in the region of the maximum pressure gradient, see Figure 7 (left) and Figure $6(\mathrm{~b})$ at $\alpha \approx 120^{\circ}$. The microgroove has negligible effect on pressure and load carrying capacity when it passes the region of cavitation.
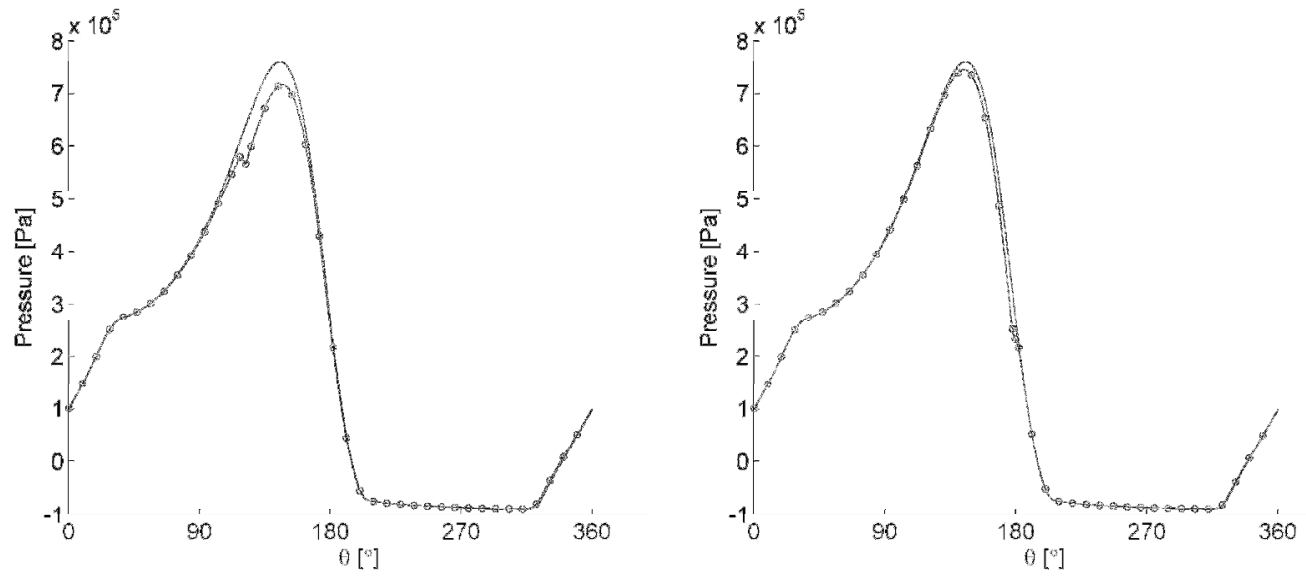

Figure 7: Pressure profiles in the midplane of the $3 D$ bearing for $\alpha=120^{\circ}$ (left) and $\alpha=$ $180^{\circ}$ (right), $d / c=1$ and $\varepsilon=0.61$. The plain curve is for the smooth shaft and the dotted curve for the shaft with a microgroove. 
Figure 6(b) also shows that the effect of the microgroove on the load carrying capacity increases with a larger depth. Load carrying capacity averaged over one revolution decreases when the microgroove depth is increased. Friction force is also decreased due to the microgroove. As a result, friction coefficient is increased for the two eccentricities considered as the loss in load carrying capacity is more pronounced than the reduction in friction force. This confirms the trend of the 2D isothermal bearing. The oscillations observed mostly around the minimum film region (Figure 6) are attributed to rapid flow variations due to the passage of the microgroove. These variations are transmitted to the local pressure and eventually causes some oscillations in load carrying capacity.

Temperature field is presented in Figure 8 in the midplane of the bearing. Lubricant is heated up by shearing action when travelling through the film. The warmest lubricant is found in the maximum film region as adiabatic conditions do not allow heat losses through the bearing surface. The coldest lubricant is seen at the oil supply groove around $\theta=30^{\circ}$. The fact that some cold lubricant is found upstream of the supply window is explained by a recirculation flow zone which drags cold lubricant upstream until the zone of film reformation, as observed by Keogh et al. (1997).

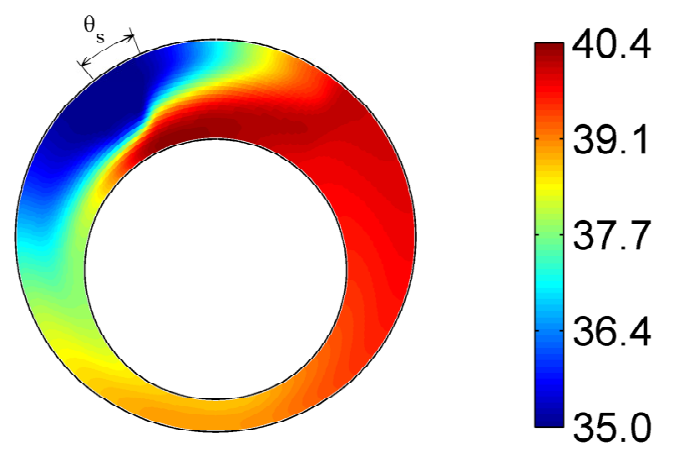

Figure 8: Temperature distribution in the midplane of the bearing with a smooth shaft for $\varepsilon$ $=0.61$. The radial clearance is magnified to make temperature field clearly visible.

Temperature at the shaft surface is presented for the textured case with $d / c=1$ in Figure 9. The lubricant inside the microgroove and/or just after its trailing edge is colder than the surrounding lubricant at the shaft surface. It can be seen that the bottom surface of the microgroove is still relatively warm at $\alpha=90^{\circ}$. While the microgroove travels towards warmer regions, the temperature of its bottom surface decreases starting at $\alpha=90^{\circ}$. Thus, a lubricant circulation and mixing occurs in the microgroove.

To investigate this process, the average temperature of the microgroove volume is presented in Figure 10. The average temperature at the corresponding projected surface for the smooth shaft is also shown for comparison. In Figure 10(a), the temperature of the shallow microgroove follows closely the temperature of the smooth shaft except in the range $100^{\circ}<\alpha$ $<250$ where colder lubricant is observed in the microgroove. The cold lubricant picked up by the microgroove is redistributed with some delay to warmer regions. In Figure 10(b), the temperature of the deeper microgroove follows a slightly different trend. When the microgroove passes over the supply window, the incoming cold lubricant penetrates further through the film thickness, pushing the warmest lubricant to the bottom of the microgroove. Therefore, lower average temperature is observed in the microgroove around this region. As the microgroove $\mathrm{d} / \mathrm{c}=1$ has a larger volume, the flow inside is subjected for a longer time to a global flow recirculation. A certain amount of lubricant constantly stays in the microgroove when global flow recirculation is encountered in it. The deeper microgroove keeps then its lowest average temperature for a longer distance than the shallow microgroove and redistributes the lubricant at a later stage. 


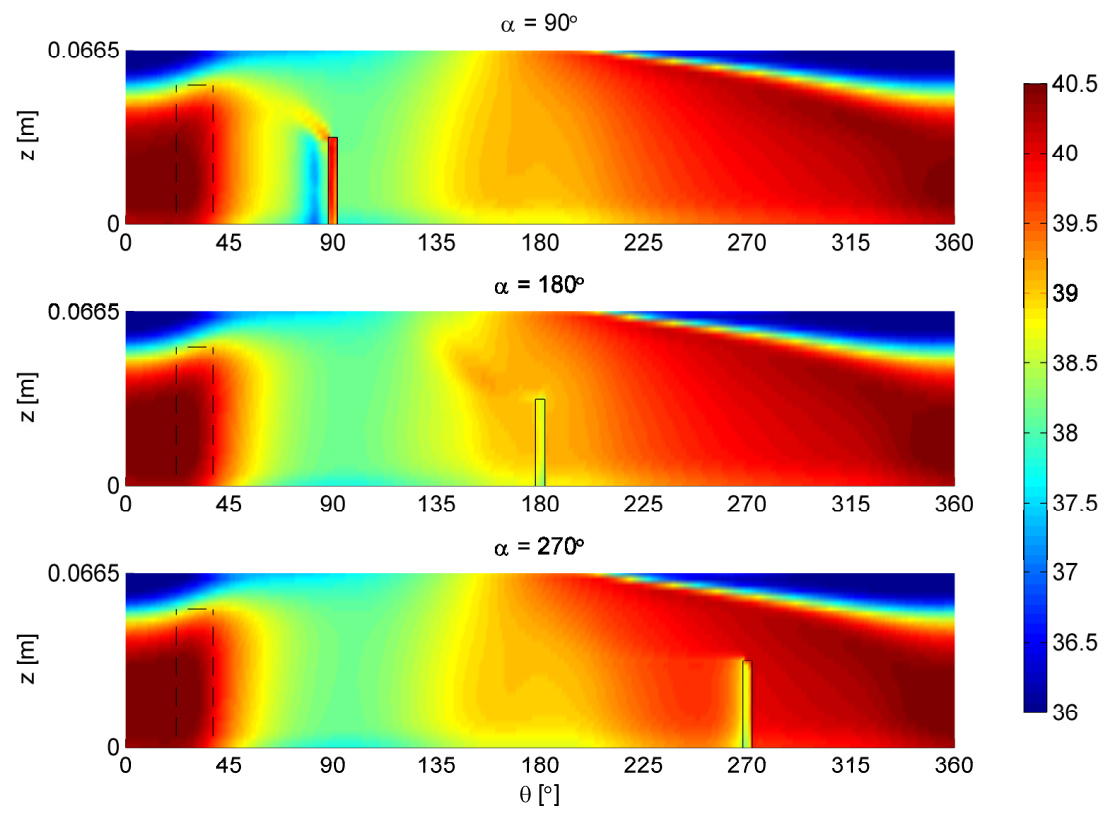

Figure 9: Temperature distribution at the shaft surface for different microgroove positions, $d / c=1$. The microgroove edges are represented by the solid lines, and the position of the lubricant supply window is shown by the dashed lines.

When global flow recirculation occurs in the microgroove, the average temperature stays almost constant in its volume as there is less exchange and mixing with the surrounding lubricant in the film. In the meantime, lubricant inside the microgroove mixes so that its temperature becomes uniform. When the recirculation disappears due to decreasing $\mathrm{d} / \mathrm{h}$ ratios at higher values of $\alpha$, the lubricant in the microgroove is mixed with the surrounding lubricant. As a consequence, the average temperature increases beyond the $2^{\text {nd }}$ dashed line in Figure 10. The deeper microgroove with $\mathrm{d} / \mathrm{c}=1$ can then cool down more efficiently the region of high temperatures as recirculating flow helps transporting a greater amount of cold lubricant. Flow recirculation inside the microgroove helps to carry colder lubricant to warmer regions. This beneficial cooling effect can prevent large drops in local viscosity.

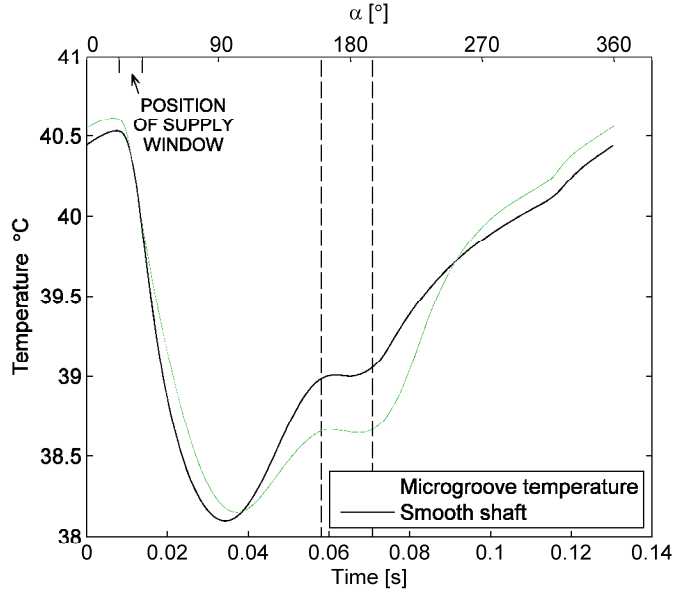

(a) $\mathrm{d} / \mathrm{c}=0.2$

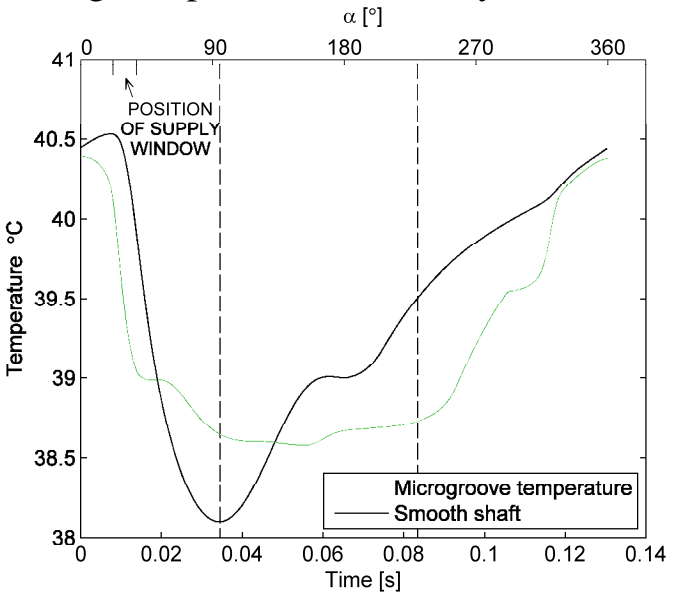

(b) $\mathrm{d} / \mathrm{c}=1$

Figure 10: Average temperature of the microgroove volume and at the corresponding projected surface for the smooth shaft, $\varepsilon=0.61$. Vertical dashed lines mark the appearance and vanishing of a global flow recirculation in the microgroove. 


\section{CONCLUSION}

A journal bearing with a microgroove on the shaft has been investigated. From this study, it has been found that:

- The grid update method, moving the mesh nodes at each time step, is reliable to simulate the motion of microtexture for both finite and infinitely wide bearings.

- A microgroove located on the shaft surface modifies pressure for both the finite and infinitely wide bearings studied, producing a local drop. The microgroove has a negligible effect on pressure and load carrying capacity when passing through the cavitation zone.

- Load carrying capacity averaged over one revolution of the bearing with a microgroove on the shaft is lower than that of the bearing with a smooth shaft. The averaged load carrying capacity is reduced when the microgroove depth increases.

- The microgroove carries and distributes a colder lubricant in the warmest regions. This effect, more pronounced with deeper microgrooves, is due to a global flow recirculation inside the microgroove, which improves mixing.

\section{ACKNOWLEDGEMENT}

The authors would like to acknowledge the financial support provided by the Swedish Research Council (Vetenskapsrådet). The research presented was carried out as a part of the "Swedish Hydropower Centre - SVC". SVC has been established by the Swedish Energy Agency, Elforsk and Svenska Kraftnät together with Luleå University of Technology, The Royal Institute of Technology, Chalmers University of Technology and Uppsala University (www.svc.nu).

\section{REFERENCES}

Cupillard, S., Cervantes, M.J., and Glavatskih, S., Pressure build-up mechanism in a textured inlet of a hydrodynamic contact, Journal of Tribology, 130. 021701-1-10, 2008a.

Cupillard, S., Cervantes, M.J., and Glavatskih, S., A CFD study of a finite textured journal bearing, In 24th Symposium on Hydraulic Machines and Systems (IAHR), Foz do Iguassu, Brazil, 2008b.

Jang, G.H., and Chang, D.I., Analysis of a hydrodynamic herringbone grooved journal bearing considering cavitation, Journal of Tribology, 122(1), pp. 103-109, 2000.

Jourak, A., Cervantes, M.J., Cupillard, S., and Glavatskih, S., CFD analysis of a journal bearing with a microgroove on the shaft, In The 19th International Symposium on Transport Phenomena, Reykjavik, Iceland, 2008.

Keogh, P.S., Gomiciaga, R., and Khonsari, M.M., CFD based design techniques for thermal prediction in a generic two-axial groove hydrodynamic journal bearing, Journal of Tribology, Transactions of the ASME, 119(3), pp. 428-436, 1997.

Kobayashi, T., Numerical analysis of herringbone-grooved gas-lubricated journal bearings using a multigrid technique, Journal of Tribology, 121(1), pp. 148-156, 1999.

Snegovskii, F.P., and Bulyuk, N.G., Study of lubrication of sliding bearings with microgrooves on the shafts, Trenie i Iznos, 4(2), pp. 322-329, 1983.

Winoto, S.H., Hou, Z.Q., Ong, S.K., Rondonuwu, C.C., and Zhang, Q.D., Effects of herringbone groove patterns on performance of vertical hydrodynamic journal bearings, Tribology Transactions, 45(3), pp. 318-323, 2002. 